\title{
The metallicities of UM 151, UM 408 and A 1228+12 revisited
}

\author{
S. A. Pustilnik ${ }^{1,4}$, A. Y. Kniazev ${ }^{1,2,4}$, J. Masegosa ${ }^{3 \star}$, I. Márquez ${ }^{3 *}$, \\ A. G. Pramskij ${ }^{1,4}$, and A. V. Ugryumov ${ }^{1,4}$
}

1 Special Astrophysical Observatory RAS, Nizhnij Arkhyz, Karachai-Circassia, 369167 Russia

2 Max Planck Institut für Astronomie, Königstuhl 17, 69117, Heidelberg, Germany

3 Instituto de Astrofísica de Andalucía, Granada, Spain

4 Isaac Newton Institute of Chile, SAO Branch

Received 5 December 2001 / Accepted 16 April 2002

\begin{abstract}
We present the results of new spectrophotometry and heavy element abundance determinations for 3 dwarf galaxies UM 151, UM 408 and A 1228+12 (RMB 132). These galaxies have been claimed in the literature to have very low metallicities, corresponding to $\log (\mathrm{O} / \mathrm{H})+12 \leq 7.65$, that are in the metallicity range of some candidate local young galaxies. We present higher S/N data for these three galaxies. UM 151 and UM 408 have significantly larger metallicities: $\log (\mathrm{O} / \mathrm{H})+12=8.5$ and 7.93, respectively. For A $1228+12$ our new $\log (\mathrm{O} / \mathrm{H})+12=$ 7.73 is close to that recalculated from earlier data (7.68). Thus, the rederived metallicities allow us to remove these objects from the list of galaxies with $Z \lesssim 1 / 20 Z_{\odot}$.
\end{abstract}

Key words. galaxies: dwarf - galaxies: stellar content - galaxies: abundances galaxies: individual: UM 151=Mkn 1169, UM 408, A 1228+12=RMB 132

\section{Introduction}

Extremely metal-deficient gas-rich galaxies (commonly taken as objects with an ionized gas metallicity $Z \lesssim$ $1 / 20 Z_{\odot}$ ) have received much attention recently. The discovery of an unusually low metallicity of the ionized gas in the blue compact galaxy (BCG) I Zw 18 (Mkn 116) (Searle \& Sargent 1972) led to the claim that such objects can be considered as the best candidates for truly local young galaxies. However, the search for similar galaxies conducted by several groups (e.g., Sargent \& Kunth 1986; Terlevich et al. 1991) has failed to find a large population with such extremely low metallicities. In their review, Kunth \& Östlin (2000) summarize and discuss many problems related to very metal-poor galaxies and they present a compilation of all the known gas-rich galaxies with $Z \lesssim 1 / 20 Z_{\odot}$. From the analysis of the ratios of $\mathrm{C} / \mathrm{O}$ and N/O abundances Izotov \& Thuan (1999) suggested that the lowest metallicity blue compact galaxies should experience their first episode of star formation (SF) and therefore the hypothesis of youth for these galaxies needs to be carefully studied. In any case the properties of such

Send offprint requests to: S. Pustilnik, e-mail: sap@sao.ru

* Visiting Astronomer, German-Spanish Astronomical Center, Calar Alto, operated by the Max-Planck-Institut für Astronomie jointly with the Spanish National Commission for Astronomy. galaxies best approximate those of young low-mass galaxies formed at the epoch of galaxy formation.

Therefore, very metal-poor galaxies in the Local Universe attract much attention, and multiwavelength studies of such objects have been performed to better identify their nature. However not all such galaxies found in the early studies as very metal-deficient (Kunth \& Östlin 2000) have spectroscopic data of sufficient quality for an accurate determination of the metallicity. Since detailed studies of individual galaxies require significant effort and observational time, thus, one should be confident about the very low metallicity of the selected galaxies.

In Paper I (Kniazev et al. 2001a) we reported a revision of 3 BCGs from the list of Kunth \& Östlin (2000). For one, the low metallicity value was confirmed while the other two showed an underestimation of 0.4 dex. In this paper we present high $\mathrm{S} / \mathrm{N}$ spectroscopy of two more BCGs (UM 408 and A 1228+12) from the list by Kunth \& Östlin (2000), for which the metallicity determination raised some concerns, and one galaxy (UM 151) from the work by Telles (1996). All these galaxies were claimed as $Z \sim 1 / 20 Z_{\odot}$ objects based on the results of earlier spectroscopy. As a result of the previous and present work the number of extremely metal-deficient BCGs from the Kunth \& Östlin list has decreased by $\sim 20 \%$. The remaining objects are in fact representative of very metal-deficient BCGs and deserve detailed multiwavelength studies. 
Table 1. Journal of observations.

\begin{tabular}{|c|c|c|c|c|c|c|c|}
\hline $\begin{array}{l}\text { Object } \\
(1) \\
\end{array}$ & $\begin{array}{l}\text { Date } \\
(2) \\
\end{array}$ & $\begin{array}{c}\text { Exposure } \\
\text { time }[\mathrm{s}] \\
(3) \\
\end{array}$ & $\begin{array}{c}\text { Wavelength } \\
\text { Range }[\AA] \\
(4)\end{array}$ & $\begin{array}{c}\text { Dispersion } \\
{[\AA / \text { A pixel }]} \\
(5) \\
\end{array}$ & $\begin{array}{c}\text { Seeing } \\
\text { [arcsec] } \\
(6) \\
\end{array}$ & $\begin{array}{c}\text { Airmass } \\
(7) \\
\end{array}$ & $\begin{array}{c}\text { PA } \\
\text { [degree] } \\
(8) \\
\end{array}$ \\
\hline UM 151 & 1.02 .2000 & $2 \times 1800$ & $3700 \div 7000$ & $0.81 / 0.54$ & 1.8 & 1.50 & 0 \\
\hline UM 408 & 2.02.2000 & $2 \times 1800$ & $3700 \div 7000$ & $0.81 / 0.54$ & 1.4 & 1.30 & 6 \\
\hline A $1228+12$ & 20.01.2001 & $2 \times 1800$ & $3700 \div 7400$ & 2.4 & 1.7 & 1.18 & 74 \\
\hline
\end{tabular}

\section{Observations and reduction}

\subsection{Observations}

The spectra of UM 151 and UM 408 were obtained with the TWIN spectrograph attached to the Cassegrain focus of the $3.5 \mathrm{~m}$ telescope at the Calar Alto Observatory (Spain) as supplementary objects to the main program devoted to the detailed spectroscopy of the HSS (Hamburg/SAO Survey, Ugryumov et al. 2001, and references therein) blue compact galaxies. Parameters of these observations are shown in Table 1. The setup used for TWIN was the T07 grating in second order for the blue and T06 in first order for the red arm, that provided dispersions of $54 \AA \mathrm{mm}^{-1}$ and $36 \AA \mathrm{mm}^{-1}$ respectively. We have used the CCD detectors SITE12a-11 and SITe6a11 for the blue and red arms with the $5500 \AA$ beam splitter and a slit width of 2 ". 1 for UM 151 and 1 ". 2 for UM 408. The resulting FWHM spectral resolution measured on strong lines were $3.1 \AA$ and $2.5 \AA$ in the blue and red, for UM 151, and 2.9 and $2.6 \AA$, for UM 408. The scale along the slit was $0 . .56 \mathrm{pix}^{-1}$.

The spectroscopic data for A $1228+12$ (RMB 132) were obtained with the $6 \mathrm{~m}$ telescope of the Special Astrophysical Observatory of the Russian Academy of Sciences (SAO RAS). Parameters of these observations are shown in Table 1. The long-slit spectrograph (LSS) (Afanasiev et al. 1995) was used with a Photometrics CCD detector of $1024 \times 1024$ pixels with a $24 \times 24 \mu \mathrm{m}$ pixel size. Observations were conducted mainly with the software package NICE in MIDAS, as described by Kniazev \& Shergin (1995). The scale along the slit was $00^{\prime \prime} 39 \mathrm{pix}^{-1}$. A grating with 651 grooves $\mathrm{mm}^{-1}$ and a slit width of $2^{\prime \prime}$ were used, giving a $F W H M$ spectral resolution of $8 \AA$. Two 0.5-hour spectra were obtained, one after the other, each in the spectral range of $3700-6100 \AA$ and $5000-7400 \AA$, with the same pointing and long slit orientation. Spectra were extracted from the same region and the two spectra were combined to get the full spectrum of the object for analysis.

For each night we obtained biases, flat-fields and illumination correction images. Comparison lamps of $\mathrm{Fe}-\mathrm{Ne}$ and Ar-Ne-He were used for wavelength calibration for the Calar Alto and SAO data, respectively. For flux calibrations, spectrophotometric standard stars from Bohlin (1996) for the $6 \mathrm{~m}$ telescope observations and Oke (1990) for the $3.5 \mathrm{~m}$ telescope were used. Average sensitivity curves were produced for each night with rms deviations of $\sim 5 \%$ in the whole spectral blue + red range.

\subsection{Reduction}

Standard reduction procedures were used with the $\operatorname{IRAF}^{1}$ package. Once 2D spectra were wavelength calibrated and sky subtracted, flux calibration was performed by using the average sensitivity curves.

The 1D spectra were extracted with the apertures of $3.6 \times 2.0^{\prime \prime}, 6.2 \times 2.1^{\prime \prime}$ and $6.2 \times 2.1^{\prime \prime}$, respectively for the galaxies A 1228+12, UM 151 and UM 408. The 1D final spectra are shown in Fig. 1. The continuum determination and the measurements of the flux and equivalent width $(E W)$ of spectral lines were performed with MIDAS $^{2}$ (for details, see e.g., Kniazev et al. 2000). EWs for individual emission lines were measured with the standard MIDAS procedure INTEGRATE/LINE. The flux and equivalent width of the blended lines were measured using Gaussian decomposition fitting. In both cases the background was drawn by two methods: manually and with the use of the automatic procedure, with the help of the algorithm, described in detail by Shergin et al. (1996). The results of both cases were compared. The errors of the sensitivity curve and those of the line intensities have been combined in quadrature and propagated to calculate element abundances.

In particular, for the A $1228+12$ spectrum with $\sim 7 \AA$ resolution, deblending was performed for $\mathrm{H} \gamma /[\mathrm{O}$ III $] \lambda 4363$, $\mathrm{H} \alpha /[\mathrm{N}$ II $] \lambda 6548,6584$, [O I $] \lambda 6300 /[\mathrm{S}$ II $] \lambda 6312$. With these procedures the redshift and the line width were derived first for the stronger line of the blend. For the Gaussian fitting of the fainter blend components, these parameters have been fixed with the values derived for the stronger component. For the fitting of $[\mathrm{N}$ II $] \lambda 6548,6584$ lines we also fixed the intensity ratio of the two lines as $1: 3$, expected from theory (e.g. Aller 1984). The uncertainties of these fitted values were determined from the residual noise of the spectrum near the lines under analysis.

${ }^{1}$ IRAF: the Image Reduction and Analysis Facility is distributed by the National Optical Astronomy Observatories, which is operated by the Association of Universities for Research in Astronomy, In. (AURA) under cooperative agreement with the National Science Foundation (NSF).

2 MIDAS is an acronym for the European Southern Observatory package - Munich Image Data Analysis System. 

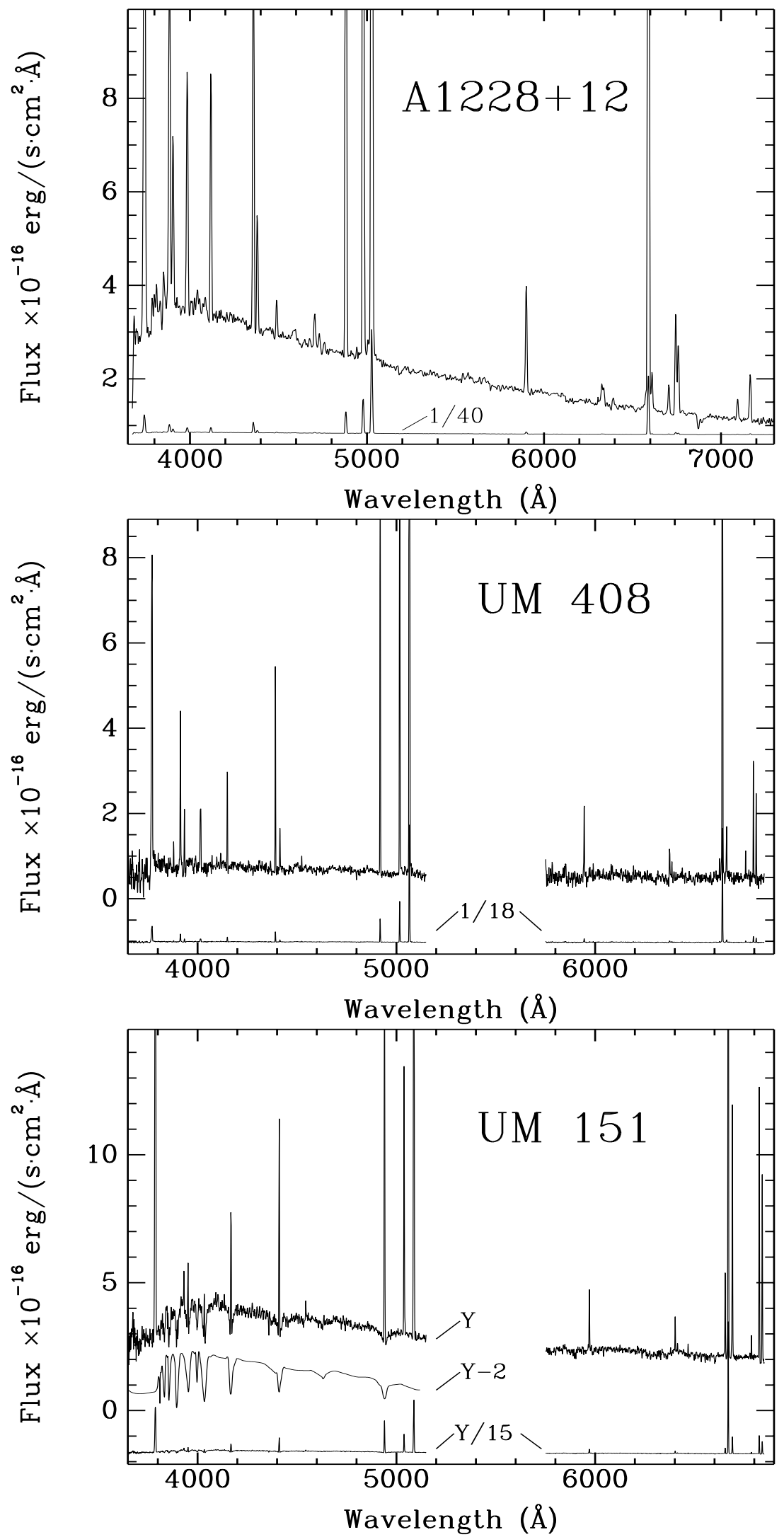

Fig. 1. 1D-spectra in the observed wavelength scale for the three galaxies discussed in the paper. In the bottom of each spectra the scaled down versions are drawn to show the relative intensities of strong lines. For UM 151 the continuum with Balmer absorption lines is shown separately, shifted down along the ordinate $(Y)$ axis by two flux units (marked as " $Y-2$ "). 
Table 2. Line intensities of the studied galaxies.

\begin{tabular}{|c|c|c|c|c|c|c|}
\hline \multirow[b]{2}{*}{$\lambda_{0}(\AA) \operatorname{Ion}^{a}$} & \multicolumn{2}{|c|}{ A $1228+12$} & \multicolumn{2}{|c|}{ UM 151} & \multicolumn{2}{|c|}{ UM 408} \\
\hline & $F(\lambda) / F(\mathrm{H} \beta)^{b}$ & $I(\lambda) / I(\mathrm{H} \beta)^{c}$ & $F(\lambda) / F(\mathrm{H} \beta)^{b}$ & $I(\lambda) / I(\mathrm{H} \beta)^{c}$ & $F(\lambda) / F(\mathrm{H} \beta)^{b}$ & $I(\lambda) / I(\mathrm{H} \beta)^{c}$ \\
\hline $3727[\mathrm{O}$ II] & $0.9503 \pm 0.0722$ & $0.9259 \pm 0.0778$ & $2.4257 \pm 0.1997$ & $2.6919 \pm 0.2466$ & $1.4502 \pm 0.1280$ & $2.1700 \pm 0.2045$ \\
\hline 3835 H9 & - & - & - & - & $0.0590 \pm 0.0187$ & $0.0860 \pm 0.0380$ \\
\hline 3868 [Ne III $]$ & $0.3791 \pm 0.0308$ & $0.3693 \pm 0.0324$ & - & - & $0.3812 \pm 0.0409$ & $0.5390 \pm 0.0601$ \\
\hline $3889 \mathrm{He} \mathrm{I}+\mathrm{H} 8$ & $0.1634 \pm 0.0170$ & $0.1958 \pm 0.0275$ & $0.1262 \pm 0.0259$ & $0.1815 \pm 0.0528$ & $0.1474 \pm 0.0246$ & $0.2082 \pm 0.0434$ \\
\hline $3967[\mathrm{Ne}$ III $]+\mathrm{H} 7$ & $0.2254 \pm 0.0196$ & $0.2555 \pm 0.0288$ & $0.1020 \pm 0.0285$ & $0.1470 \pm 0.0527$ & $0.2077 \pm 0.0294$ & $0.2842 \pm 0.0507$ \\
\hline $4101 \mathrm{H} \delta$ & $0.2210 \pm 0.0196$ & $0.2492 \pm 0.0279$ & $0.2409 \pm 0.0320$ & $0.2947 \pm 0.0518$ & $0.2113 \pm 0.0230$ & $0.2749 \pm 0.0425$ \\
\hline $4340 \mathrm{H} \gamma$ & $0.4605 \pm 0.0360$ & $0.4798 \pm 0.0416$ & $0.4086 \pm 0.0389$ & $0.4588 \pm 0.0553$ & $0.4251 \pm 0.0362$ & $0.5056 \pm 0.0492$ \\
\hline 4363 [O III] & $0.1055 \pm 0.0130$ & $0.1028 \pm 0.0131$ & - & - & $0.0851 \pm 0.0170$ & $0.1002 \pm 0.0202$ \\
\hline $4471 \mathrm{He} \mathrm{I}$ & $0.0321 \pm 0.0073$ & $0.0313 \pm 0.0073$ & $0.0271 \pm 0.0144$ & $0.0273 \pm 0.0151$ & $0.0266 \pm 0.0113$ & $0.0302 \pm 0.0129$ \\
\hline 4686 He II & $0.0403 \pm 0.0078$ & $0.0392 \pm 0.0078$ & - & - & - & - \\
\hline $4713[$ Ar IV $]+$ He I & $0.0208 \pm 0.0073$ & $0.0203 \pm 0.0073$ & - & - & - & - \\
\hline $4713[$ Ar IV $]$ & $0.0163 \pm 0.0080$ & $0.0159 \pm 0.0080$ & - & - & - & - \\
\hline $4861 \mathrm{H} \beta$ & $1.0000 \pm 0.0771$ & $1.0000 \pm 0.0800$ & $1.0000 \pm 0.0829$ & $1.0000 \pm 0.0899$ & $1.0000 \pm 0.0758$ & $1.0000 \pm 0.0789$ \\
\hline 4959 [O III] & $1.5651 \pm 0.1213$ & $1.5251 \pm 0.1213$ & $0.5509 \pm 0.0493$ & $0.5270 \pm 0.0490$ & $1.7714 \pm 0.1318$ & $1.7174 \pm 0.1291$ \\
\hline 5007 [O III] & $4.6938 \pm 0.3588$ & $4.5736 \pm 0.3590$ & $1.6881 \pm 0.1343$ & $1.6069 \pm 0.1328$ & $5.6848 \pm 0.4445$ & $5.4322 \pm 0.4294$ \\
\hline 5876 He I & $0.0992 \pm 0.0107$ & $0.0967 \pm 0.0109$ & $0.1227 \pm 0.0211$ & $0.1077 \pm 0.0194$ & $0.1538 \pm 0.0311$ & $0.1161 \pm 0.0238$ \\
\hline $6300[\mathrm{O}$ I $]$ & $0.0230 \pm 0.0096$ & $0.0224 \pm 0.0096$ & $0.0636 \pm 0.0171$ & $0.0540 \pm 0.0152$ & $0.0584 \pm 0.0232$ & $0.0399 \pm 0.0161$ \\
\hline 6312 [S III] & $0.0146 \pm 0.0087$ & $0.0143 \pm 0.0087$ & $0.0161 \pm 0.0119$ & $0.0137 \pm 0.0105$ & $0.0309 \pm 0.0231$ & $0.0211 \pm 0.0159$ \\
\hline $6364[\mathrm{O}$ I] & $0.0085 \pm 0.0055$ & $0.0083 \pm 0.0055$ & $0.0203 \pm 0.0145$ & $0.0171 \pm 0.0128$ & - & - \\
\hline $6548[\mathrm{~N} \mathrm{II}]$ & $0.0102 \pm 0.0068$ & $0.0100 \pm 0.0068$ & $0.1455 \pm 0.0234$ & $0.1212 \pm 0.0206$ & $0.0342 \pm 0.0306$ & $0.0222 \pm 0.0201$ \\
\hline $6563 \mathrm{H} \alpha$ & $2.6763 \pm 0.2002$ & $2.6217 \pm 0.2185$ & $3.4333 \pm 0.2673$ & $2.8819 \pm 0.2533$ & $4.3736 \pm 0.3226$ & $2.8264 \pm 0.2289$ \\
\hline $6584[\mathrm{~N} \mathrm{II}]$ & $0.0310 \pm 0.0229$ & $0.0302 \pm 0.0229$ & $0.4404 \pm 0.0383$ & $0.3661 \pm 0.0353$ & $0.0957 \pm 0.0245$ & $0.0615 \pm 0.0160$ \\
\hline 6678 He I & $0.0250 \pm 0.0071$ & $0.0244 \pm 0.0071$ & $0.0379 \pm 0.0144$ & $0.0313 \pm 0.0124$ & $0.0506 \pm 0.0220$ & $0.0319 \pm 0.0140$ \\
\hline 6717 [S II] & $0.0936 \pm 0.0109$ & $0.0912 \pm 0.0113$ & $0.4766 \pm 0.0409$ & $0.3926 \pm 0.0378$ & $0.2467 \pm 0.0342$ & $0.1545 \pm 0.0222$ \\
\hline 6731 [S II] & $0.0645 \pm 0.0096$ & $0.0628 \pm 0.0099$ & $0.3423 \pm 0.0328$ & $0.2816 \pm 0.0298$ & $0.1635 \pm 0.0260$ & $0.1021 \pm 0.0168$ \\
\hline 7065 He I & $0.0173 \pm 0.0062$ & $0.0168 \pm 0.0063$ & - & - & - & - \\
\hline 7136 [Ar III] & $0.0430 \pm 0.0083$ & $0.0419 \pm 0.0085$ & - & - & - & - \\
\hline$C(\mathrm{H} \beta) \operatorname{dex}$ & $0.00 \pm$ & \pm 0.10 & $0.20=$ & \pm 0.10 & $0.57 \pm$ & \pm 0.10 \\
\hline$E W(\mathrm{abs}) \AA$ & $2.45 \pm$ & \pm 1.08 & $0.90=$ & \pm 0.67 & $0.05 \pm$ & \pm 0.94 \\
\hline$F(\mathrm{H} \beta)^{d}$ & 232 & \pm 13 & & \pm 4 & $33=$ & \pm 2 \\
\hline$E W(\mathrm{H} \beta$, emis $) \AA$ & 93 & \pm 5 & 20 & \pm 2 & $50=$ & \pm 3 \\
\hline
\end{tabular}

${ }^{a}$ The rest-frame wavelength in $\AA ;^{b}$ observed flux ratio; ${ }^{c}$ corrected flux ratio; ${ }^{d}$ in units of $10^{-16} \mathrm{ergs} \mathrm{s}^{-1} \mathrm{~cm}^{-2}$.

These uncertainties were combined, as well as for all other measured line intensities, with the other error components (see below). Therefore, the derived errors of [N II] lines can be large, and their intensity ratio in the table is theoretical. For the TWIN spectra the spectral resolution is sufficient to measure each line separately. While in the spectrum of UM 408 [N II]-lines are rather faint, their line ratio is occasionally close to the theoretical value. In addition to the noise of the underlying continuum, quoted errors in the line intensities include two more components: one comes from Poisson statistics of photon flux, the other comes from the uncertainties of the sensitivity curve, contributing a few per cent to all lines. The line intensity errors presented in Table 2 incorporate all three components, and thus should be reliable estimators for other derived physical parameters and chemical abundances in the H II regions considered. An independent check of the reliability of the cited errors is the good consistency between our results and the results of Kinman \& Davidson (1981) for A $1228+12$ (see Sect. 4.3). Both sets of line intensities are consistent within the cited errors, if their extinction correction is accounted for. The latter can be overestimated, since Kinman \& Davidson indicate a mismatch in the continuum level for independent red and blue spectra. Another factor leading to small differences is that Kinman \& Davidson did not account for the underlying Balmer absorption. In the present work we determined underlying Balmer absorption at $\mathrm{H} \beta$ of $E W(\mathrm{H} \beta) \sim$ $2.5 \AA$. We derived from the spectrum of A $1228+12$ the value of $C(\mathrm{H} \beta)=0$. This is consistent within the cited 
Table 3. Abundances in the studied galaxies.

\begin{tabular}{|c|c|c|}
\hline Value & A $1228+12$ & UM 408 \\
\hline$T_{\mathrm{e}}(\mathrm{OIII})(\mathrm{K})$ & $16100 \pm 1100$ & $14800 \pm 1400$ \\
\hline$T_{\mathrm{e}}(\mathrm{OII})(\mathrm{K})$ & $14300 \pm 900$ & $13600 \pm 1200$ \\
\hline$T_{\mathrm{e}}(\mathrm{SIII})(\mathrm{K})$ & $15100 \pm 900$ & $14000 \pm 1200$ \\
\hline$N_{\mathrm{e}}(\mathrm{SII})\left(\mathrm{cm}^{-3}\right)$ & $10 \pm 10$ & $10 \pm 10$ \\
\hline $\mathrm{O}^{+} / \mathrm{H}^{+}\left(\times 10^{5}\right)$ & $0.936 \pm 0.186$ & $2.518 \pm 0.689$ \\
\hline $\mathrm{O}^{++} / \mathrm{H}^{+}\left(\times 10^{5}\right)$ & $4.198 \pm 0.770$ & $6.050 \pm 1.546$ \\
\hline $\mathrm{O}^{+++} / \mathrm{H}^{+}\left(\times 10^{5}\right)$ & $0.254 \pm 0.098$ & - \\
\hline $\mathrm{O} / \mathrm{H}\left(\times 10^{5}\right)$ & $5.388 \pm 0.798$ & $8.568 \pm 1.693$ \\
\hline $12+\log (\mathrm{O} / \mathrm{H})$ & $7.73 \pm 0.06$ & $7.93 \pm 0.09$ \\
\hline $\mathrm{N}^{+} / \mathrm{H}^{+}\left(\times 10^{7}\right)$ & $2.470 \pm 1.472$ & $5.491 \pm 1.439$ \\
\hline$I C F(\mathrm{~N})$ & 5.758 & 3.402 \\
\hline $\log (\mathrm{N} / \mathrm{O})$ & $-1.58 \pm 0.27$ & $-1.66 \pm 0.14$ \\
\hline $\mathrm{Ne}^{++} / \mathrm{H}^{+}\left(\times 10^{5}\right)$ & $0.737 \pm 0.143$ & $1.369 \pm 0.381$ \\
\hline$I C F(\mathrm{Ne})$ & 1.284 & 1.416 \\
\hline $\log (\mathrm{Ne} / \mathrm{O})$ & $-0.76 \pm 0.11$ & $-0.65 \pm 0.15$ \\
\hline $\mathrm{S}^{+} / \mathrm{H}^{+}\left(\times 10^{7}\right)$ & $1.658 \pm 0.232$ & $2.990 \pm 0.537$ \\
\hline $\mathrm{S}^{++} / \mathrm{H}^{+}\left(\times 10^{7}\right)$ & $7.212 \pm 4.560$ & $13.490 \pm 10.660$ \\
\hline$I C F(\mathrm{~S})$ & 1.709 & 1.357 \\
\hline $\log (\mathrm{S} / \mathrm{O})$ & $-1.55 \pm 0.23$ & $-1.58 \pm 0.29$ \\
\hline $\mathrm{Ar}^{++} / \mathrm{H}^{+}\left(\times 10^{7}\right)$ & $1.500 \pm 0.327$ & - \\
\hline $\mathrm{Ar}^{+++} / \mathrm{H}^{+}\left(\times 10^{7}\right)$ & $1.957 \pm 1.023$ & 一 \\
\hline$I C F(\mathrm{Ar})$ & 1.026 & - \\
\hline $\log (\mathrm{Ar} / \mathrm{O})$ & $-2.18 \pm 0.15$ & - \\
\hline
\end{tabular}

uncertainties $\left(\sigma_{C(\mathrm{H} \beta)}=0.10\right)$ with the minimum value of $C(\mathrm{H} \beta)=0.043$ following from the Galaxy extinction in this direction, $A_{B}=0.12 \mathrm{mag}$ (see Table 4 ). We have checked the effect of the change of $C(\mathrm{H} \beta)$ from zero to 0.043 on the derived element abundances. The $\mathrm{O} / \mathrm{H}$ value does not change at all. The values of $\log (\mathrm{N} / \mathrm{O}),(\mathrm{Ne} / \mathrm{O})$, $(\mathrm{S} / \mathrm{O})$ and $(\mathrm{Ar} / \mathrm{O})$ change by only $0.02-0.03 \mathrm{dex}$, which is many times smaller than their cited uncertainties.

\section{Results of the abundance determination}

Relative intensities of all emission lines together with the equivalent width $E W(\mathrm{H} \beta$, emis), extinction coefficient $C(\mathrm{H} \beta)$ and the equivalent width of the hydrogen absorption lines are given in Table 2 . The extinction coefficient $C(\mathrm{H} \beta)$ was derived from the hydrogen Balmer emission decrement using the self-consistent method described by Izotov et al. (1994). For both UM 408 and A 1228+12 the continuum was drawn as a running mean without accounting for possible absorption features. For UM 151, before obtaining emission line intensities, the underlying continuum was drawn including Balmer absorption lines and
Table 4. Main parameters of studied galaxies.

\begin{tabular}{lccc}
\hline \hline Parameter & UM 151 & UM 408 & A 1228+12 \\
\hline$\alpha_{2000}$ & 015738.87 & 021123.55 & 123048.52 \\
$\delta_{2000}$ & +022523.9 & +022031.0 & +120242.1 \\
$A_{B}{ }^{N}$ & 0.12 & 0.15 & 0.12 \\
$B_{\text {tot }}$ & $16.21^{(1)}$ & $17.74^{(1)}$ & $17.15^{(3)}$ \\
$V_{\text {Hel }}\left(\mathrm{km} \mathrm{s}^{-1}\right)$ & $4851^{(2)}$ & $3507^{(4)}$ & $1263^{(5)}$ \\
Dist $(\mathrm{Mpc})$ & 64.7 & 46.8 & $17.0^{V}$ \\
$M_{B}^{0}(6)$ & -17.96 & -15.76 & -14.10 \\
Opt. size $\left(^{\prime \prime}\right)^{(7)}$ & $35 \times 15.3^{(1)}$ & $15.6 \times 10.6^{(4)}$ & $12 \times 9^{(3)}$ \\
Opt. size $(\mathrm{kpc})$ & $11.0 \times 2.4^{(2)}$ & $3.5 \times 2.4^{(2)}$ & $1.0 \times 0.75^{(2)}$ \\
$12+\log (\mathrm{O} / \mathrm{H})$ & $8.5^{(2)}$ & $7.93^{(2)}$ & $7.73^{(2)}$ \\
H I flux & $<1.2^{(9)}$ & $1.5^{(4)}$ & $1.4^{(5)}$ \\
$W_{50}\left(\mathrm{~km} \mathrm{~s}^{-1}\right)$ & - & $77^{(4)}$ & $84^{(5)}$ \\
$M(\mathrm{H} \mathrm{I})\left(10^{(8)} M_{\odot}\right)$ & $<11.9^{(2)}$ & $7.8^{(2,4)}$ & $0.95^{(2,5)}$ \\
$M(\mathrm{H} \mathrm{I}) / \mathrm{L}_{\mathrm{B}}{ }^{(10)}$ & $<2.0^{(2)}$ & $2.5^{(2)}$ & $1.5^{(2)}$ \\
\hline
\end{tabular}

(1) - Salzer et al. (1989b) $\left(V_{25}\right.$-isophote, $b / a-$ minor-to-major axis ratio, from LEDA).

(2) - parameters derived in this paper.

(3) - Binggeli \& Cameron (1993).

(4) - Smoker et al. (2000).

(5) - Staveley-Smith et al. (1992).

(6) - corrected for the Galaxy extinction.

(7) $-a \times b$ at $\mu_{B}=25 \mathrm{mag} / \mathrm{sq}$. arcsec. See note (1).

(8) - in units of $\left(\mathrm{Jy} \cdot \mathrm{km} \mathrm{s}^{-1}\right)$.

(9) - Thuan et al. 1999; upper limit is estimated for $W_{50}=100 \mathrm{~km} \mathrm{~s}^{-1}$.

(10) - in units of $\left(M / L_{B}\right) \odot$.

(V) - accepted for the Virgo cluster (Tikhonov et al. 2000).

(N) - data from NED, Schlegel et al. (1998).

other apparent absorption features. Their measured equivalent widths were used for age estimates (see Table 5). The quoted $E W$ (abs) for this galaxy in Table 2 is the residual value that is derived after the underlying continuum was drawn, including strong Balmer absorptions. The $E W$ of emission $\mathrm{H} \beta$ is calculated on a running mean continuum that will be compared with the model value below. The derived extinction coefficients are in the range from zero for A 1228+12, 0.2 for UM 151, to $\sim 0.6$ for UM 408. The latter value is somewhat larger than usually is observed in this type of galaxies. For A $1228+12$ and UM 408 the chemical abundances and physical parameters have been obtained with the method outlined in the paper of Izotov et al. (1997). The resulting values are given in Table 3 .

For UM 151, since no measurable [O III] $\lambda 4363 \AA$ line has been detected, the metallicity has been estimated by means of empirical methods (see more details in Sect. 4.1).

The $E W$ of emission $\mathrm{H} \beta$ presented in Table 2 were used to derive starburst ages according to the Starburst99 model (Leitherer et al. 1999) in Sects. 4.1 and 4.3. It was assumed that extinction values for the ionized gas and the young stellar clusters are similar. 
Table 5. The absorption lines $E W$ and continuum ratios of UM 151.

\begin{tabular}{lr|lr}
\hline \hline Abs. line & Value & \multicolumn{1}{|c}{ Band } & Value \\
\hline$E W\left(\mathrm{H}_{\beta}\right)$ & 5.4 & CaII-K & 2.2 \\
$E W\left(\mathrm{H}_{\gamma}\right)$ & 4.0 & $G$ band & 3.2 \\
$E W\left(\mathrm{H}_{\delta}\right)$ & 6.1 & $F_{3660} / F_{4020}$ & 0.71 \\
$E W\left(\mathrm{H}_{8}\right)$ & 7.4 & $F_{3780} / F_{4020}$ & 0.88 \\
$E W\left(\mathrm{H}_{9}\right)$ & 7.9 & $F_{4510} / F_{4020}$ & 0.83 \\
$E W\left(\mathrm{H}_{10}\right)$ & 4.0 & & \\
\hline
\end{tabular}

\section{Discussion and summary}

We discuss below the individual galaxies in more detail, based mainly on the new spectral data, and the observational data from the literature, appropriate for the present discussion. The main parameters of the studied galaxies are given in Table 4 .

\subsection{UM $151=$ Mkn 1169}

This galaxy does not look like a bona fide BCG. Its appearance resembles that of a face-on disk with somewhat disturbed outermost parts, but clearly without spiral arms. Salzer (1989a) classified it as a Dwarf Amorphous Nuclear Starburst (DANS). Its absolute magnitude quoted here (Table $4, M_{B}=-17^{\mathrm{m}} \cdot 96$ ) is within the range for DANS. A bright knot is seen near the galaxy center, but does not change the generally regular appearance of this galaxy. The low metallicity value from Telles (1996) was derived from a very low $S / N$ spectrum (see Terlevich et al. 1991).

For the metallicity estimation both the Pilyugin (2001) calibration based on the strong oxygen lines and the recently reported calibration based on the [N II] line (Denicoló et al. 2002) give very close values of $12+$ $\log (\mathrm{O} / \mathrm{H}), 8.50$ and 8.47, respectively.

Balmer absorption lines and other features with reliable detections can be used to estimate the age of starformation episodes.

The Equivalent Widths $(E W)$ of Balmer absorption lines from the underlying continuum have been calculated following the prescription in González-Delgado et al. (1999) and the results are presented in Table 5. The comparison with the González-Delgado et al. (1999) models gives an age for the starburst consistent with an instantaneous starburst $\sim 10$ Myr old. The $E W$ of $\mathrm{H} \beta$ emission is also consistent with the age of instantaneous starburst of $\sim 10$ Myr (Leitherer et al. 1999). Following Raimann et al. (2000), we also measured the $E W$ of the Ca II K-line and the $G$-band as well as the continuum flux ratios in the 3 bands (see Table 5). All but one of the parameters are consistent with a mixture of two starbursts with ages $\sim 10 \mathrm{Myr}$ and a $\sim 50 \mathrm{Myr}$. The rather high $E W$ of the $G$-band suggests an additional contribution of a stellar population with an age of a few hundred Myr.
The known upper limit on Hi flux of this galaxy (see Table 4) is rather high, so the upper limit on the ratio $M(\mathrm{HI}) / L_{B}$ is consistent with the range typical of gas-rich starbursting galaxies.

\subsection{UM 408}

Appearing like a typical blue compact dwarf, this galaxy was classified by Salzer (1989a) as a Dwarf H II Hotspot Galaxy. The absolute $B$-band luminosity is $M_{B}=-15.16$ (Campos-Aguilar et al. 1993), almost at the lower end of the BCG luminosity distribution, with a quoted diameter of $2.1 \mathrm{kpc}$. The calculated metallicity reported by Masegosa et al. (1994) was $12+\log (\mathrm{O} / \mathrm{H})=7.63$ using the data from the Spectroscopic Catalogue of H II Galaxies (Terlevich et al. 1991). Using the same set of data Telles (1996) reported a value of $12+\log (\mathrm{O} / \mathrm{H})=7.66$. The new estimation with the present data suggests a significantly higher metallicity with a difference of 0.2 to 0.3 dex. This difference cannot be attributed to the reddening estimation or differences in slit positioning. Comparing the present data with Masegosa et al. (1994) and Telles (1996), the reddening coefficients are similar within the uncertainties and the values of both $E W(\mathrm{H} \beta$, emis $)$ and integrated $\mathrm{H} \beta$ flux are the same. Therefore the main reason for the discrepancy must be a poor estimation of the [O III] $\lambda 4363$ due to low $S / N$. The comparison between the line intensities of both sets of data shows that a large difference is found not only in the faint [O III] line, but also in [O II] being larger by a factor of 2 for the TWIN data. The same is also true for the measured $[\mathrm{N}$ II] line. The low $\mathrm{S} / \mathrm{N}$ on the continuum for this faint galaxy can account for the large difference in oxygen abundance.

The measured H I flux of this BCG (see Table 4) corresponds to a very high value of the parameter $M(\mathrm{HI}) / L_{B}$. The $M(\mathrm{HI}) / L_{B}$ is comparable to values derived for the most extreme objects in the sample of dwarf galaxies with extended H I (van Zee et al. 1995).

\section{3. $A 1228+12=R M B 132=$ VCC 1313}

This galaxy is one of the most compact, almost starlike in appearance, of the BCG family (Drinkwater \& Hardy 1991). It resides in the Virgo cluster, and because of the surrounding environment, its properties are probably somewhat affected by more frequent interactions with surrounding galaxies and the hot intracluster medium (ICM). There is a number of sufficiently massive candidate galaxies in the vicinity of this BCG, which could trigger its current SF burst, including NGC 4478 at the projected distance of $18.7^{\prime}$ ( $\left.\sim 90 \mathrm{kpc}\right)$ and M 87 at $20.8^{\prime}(\sim 100 \mathrm{kpc})$, whose relative radial velocities are lower than $100 \mathrm{~km} \mathrm{~s}^{-1}$.

Of the three galaxies studied in this paper, this is the faintest system with an absolute $B$ magnitude of -14.1. Taking into account its compactness and luminosity, this is the type of galaxy classified as a SearleSargent object by Salzer (1989a), or HII galaxies by 
Campos-Aguilar et al. (1993). This galaxy was one of the first BCGs studied for metallicity purposes (Kinman \& Davidson 1981, hereafter KD81). From this study the metallicity reported of $12+\log (\mathrm{O} / \mathrm{H})=7.64 \pm 0.07$ is in reasonable agreement with the present value of $7.73 \pm 0.06$, based on the new, higher $\mathrm{S} / \mathrm{N}$ data from the $6 \mathrm{~m}$ telescope. The agreement can be considered as a good one, taking into account that the areas sampled are $29 \operatorname{arcsec}^{2}$ for KD81 and $7.2 \operatorname{arcsec}^{2}$ in this work.

However, it is evident that the abundance calculations by KD81 could have some systematic differences, since these authors did not account for temperature gradients. They used one temperature for all zones, and noticed that this would lead to slight underestimation of oxygen and neon abundances. Therefore we recalculated oxygen and neon abundances from their relative intensities and the cited errors using our methodology. This resulted in an upward shift of 0.04 dex for the KD81 oxygen abundance, giving $12+\log (\mathrm{O} / \mathrm{H})=7.68 \pm 0.10$ and $\log (\mathrm{Ne} / \mathrm{O})=$ -0.75 . The difference between the KD81 O/H abundance and the recalculated one appears to be from a somewhat larger abundance of $\mathrm{O}^{+}$ions: $12+\log \left(\mathrm{O}^{+} / \mathrm{H}\right)=6.87$ from $\mathrm{KD} 81$, and 7.05 in the model with the lower $T\left(\mathrm{O}^{+}\right)$.

One of the interesting features in the spectrum of A $1228+12$ is the appearance of an emission line centered at $\lambda 4591 \AA$, with flux $\sim(2.5 \pm 1.2) \%$ of $\mathrm{H} \beta$ and $F W H M=28 \AA$. The only reasonable identification is as the Si III $\lambda 4565$ line, characteristic of WR stars. The implied radial velocity of this feature is then $\sim 450 \mathrm{~km} \mathrm{~s}^{-1}$ higher than the system velocity of the galaxy found in other (narrow) emission lines. Given the large line width and low $S / N$ ratio, the velocity shift is likely not significant. However the appearance of WR stars in the starburst region of this BCG would not be unexpected. Indeed, the observed $E W$ of $\mathrm{H} \beta$ (93 $\AA$ ) according to Fig. 85e (corresponding to metallicity $z=0.001$, the closest to the oxygen abundance of A 1228+12) from Starburst99 (Leitherer et al. 1999), corresponds to an instantaneous starburst of age 3-3.5 Myr. We adopted the Salpeter IMF with $M_{\text {low }}=0.1 M_{\odot}$ for this estimate. For the metallicity of $z=0.001$ this is exactly the age range where the models predict significant numbers of WR-stars (0.5 to $2.5 \%$ of the number of O-stars, see Schaerer \& Vacca 1998). Since we did not detect other characteristic WR features (N III/N IV $\lambda 4640$ and broad component of He II $\lambda 4686$ ), that should have comparable $E W$, doubt about the reality of the Si III $\lambda 4565$ line remains. Deeper spectroscopy is necessary in order to measure the strength of the probable WR features in this BCG.

The $M(\mathrm{HI}) / L_{B}$ ratio for this BCG is quite large (see Table 4). Accounting for very large $E W$ of strong emission lines and respective significant brightening $\left(\Delta B \gtrsim 1{ }^{\mathrm{m}} 0\right)$, this implies a large gas mass-fraction. The latter is difficult to understand for a low-mass galaxy affected both by the ICM ram pressure (unless the BCG is only now entering the hot gas) and tidal interactions from massive neighbours.
In fact, the very existence of such a low-metallicity BCG in a dense environment such as the Virgo Cluster (despite the opposite tendency of additional enrichment by heavy elements for the Virgo cluster BCGs, noticed by Izotov \& Guseva 1989), poses interesting questions on the evolutionary history of this and similar objects. It is worth mentioning that there are two more BCGs with well determined low metallicities $\left(Z \sim Z_{\odot} / 20\right)$ probably belonging to the Virgo Cluster or its outskirts: the Optical Counterpart of HI 1225+01 (Salzer et al. 1991) and VV $432=\mathrm{IC} 3105=\mathrm{VCC} 241$ (Zasov et al. 2000). Less intriguing, but also not well understood, is the appearance of SBS 0335-052 $(\mathrm{E}+\mathrm{W})$, the pair of extremely metal-deficient BCGs, situated at the outskirts of the loose galaxy group LGG 103 (Pustilnik et al. 2001; Peebles 2001).

\subsection{Conclusions}

We can summarize the results as follows:

- New high $S / N$ spectra of galaxies UM 151, UM 408 and A $1228+12$ have been presented and their metallicities revisited. For A $1228+12$ the new value is a bit larger, but consistent within the uncertainties with previous data. For the other two galaxies, significantly higher metallicities have been estimated ( $5 \sigma$ and $3 \sigma$, respectively, upward). None of the three galaxies can be included in the sample of extremely low-metallicity BCGs $\left(Z \lesssim 1 / 20 Z_{\odot}\right)$.

- More precise abundances of nitrogen, neon and sulfur are derived for UM 408 and A 1228+12.

Results from this paper and the previous work by Kniazev et al. (2001a) showed an upward revision of metallicity in some of the most metal-poor galaxies known. High $S / N$ data of a statistically significant galaxy sample are a prerequisite for studying the group properties of these intriguing objects.

Acknowledgements. We acknowledge the partial support from INTAS grant 96-0500 and Russian state program "Astronomy". The authors thank Y.Izotov for his help in TWIN data reduction and H.Lee for useful comments, suggestions and English corrections. The authors are grateful to the referee C.Leitherer for constructive criticism and useful suggestions, which helped to improve the paper. S.A.P. acknowledges the financial support from the Junta de Andalucía for a visit to the Instituto de Astrofísica de Andalucía, where part of the work on the paper was performed. J.M. and I.M. acknowledge financial support by the Spanish DGICYT, under the programs PB98-0521 and AYA2001-2089. This research has made use of the NASA/IPAC Extragalactic Database (NED) which is operated by the Jet Propulsion Laboratory, California Institute of Technology, under contract with the National Aeronautics and Space Administration. The use of the Digitized Sky Survey (DSS-II) and the APM Database (IoA, Cambridge) is gratefully acknowledged. 


\section{References}

Afanasiev, V. L., Burenkov, A. N., Vlasyuk, V. V., \& Drabek, S. V. 1995, SAO RAS internal rep., 234

Aller, L. H. 1984, Physics of Thermal Gaseous Nebulae (Dordrecht: Reidel)

Binggeli, B., \& Cameron, L. M. 1993, A\&AS, 98, 297

Bohlin, R. C. 1996, AJ, 111, 1743

Campos-Aguilar, A., Masegosa, J., \& Moles, M. 1993, AJ, 106, 1784

Denicoló, G., Terlevich, R., \& Terlevich, E. 2002, MNRAS, 330, 69

Drinkwater, M., \& Hardy, E. A. 1991, AJ, 101, 94

González Delgado, R. M., Leitherer, C., \& Heckman, T. M. 1999, ApJS, 125, 489

Izotov, Y. I., \& Guseva, N. G. 1989, Astrofizika, 30, 564

Izotov, Y. I., Thuan, T. X., \& Lipovetsky, V. A. 1994, ApJ, 435,647

Izotov, Y. I., Lipovetsky, V. A., Chaffee, F., et al. 1997, ApJ, 476, 298

Izotov, Y. I., \& Thuan, T. X. 1999, ApJ, 511, 639

Kinman, T. D., \& Davidson, K. 1981, ApJ, 243, 127

Kniazev, A. Y., \& Shergin, V. S. 1995, SAO RAS internal rep., 249,1

Kniazev, A., Pustilnik, S., Masegosa, J., et al. 2000, A\&A, 357, 101

Kniazev, A. Y., Pustilnik, S. A., Pramsky, A. G., \& Ugryumov, A. V. 2001a, A\&A, 371, 404

Kunth, D., \& Östlin, G. 2000, A\&AR, 10, 1

Leitherer, C., Schaerer, D., Goldader, J. D., et al. 1999, ApJS, 123,3

Masegosa, J., Moles, M., \& Campos-Aguilar, A. 1994, ApJ, 420,576

Östlin, G. 2000, ApJ, 535, L99

Östlin, G., \& Kunth, D. 2001, A\&A, 371, 429

Peebles, P. J. E. 2001, ApJ, 557, 495

Pilyugin, L. S. 2001, A\&A, 374, 412
Pustilnik, S. A., Brinks, E., Thuan, T. X., Lipovetsky, V. A., \& Izotov, Y. I. 2001, AJ, 121, 1413

Raimann, D., Bica, E., Storchi-Bergmann, T., Melnick, J., \& Schmitt, H. 2000, MNRAS, 314, 295

Salzer, J. J. 1989, ApJ, 347, 152

Salzer, J. J., MacAlpine, G. M., \& Boroson, T. A. 1989, ApJS, 70,447

Salzer, J. J., di Serego Alighieri, S., Matteucci, F., Giovanelli, R., \& Haynes, M. P. 1991, AJ, 101, 1258

Schlegel, D. J., Finkbeiner, D. P., \& Douglas, M. 1998, ApJ, 500,525

Sargent, W. L. W., \& Kunth, D. 1986, ApJ, 300, 496

Schaerer, D., \& Vacca, W. D. 1998, ApJ, 497, 618

Searle, L., \& Sargent, W. L. W. 1972, ApJ, 173, 25

Shergin, V. S., Kniazev, A. Y., \& Lipovetsky, V. A. 1996, Astronomische Nachrichten, 317, 95

Skillman, E. D., Kennicutt, R. C., \& Hodge, P. W. 1989, ApJ, 347,875

Smoker, J. V., Davies, R. D., Axon, D. J., \& Hummel, F. 2000, MNRAS, 361, 19

Staveley-Smith, L., Davies, R. D., \& Kinman, T. D. 1992, MNRAS, 258, 334

Telles, E. 1996, Ph.D. Thesis, Institute of Astronomy, Cambridge

Terlevich, R., Melnick, J., Masegosa, J., Moles, M., \& Copetti, M. V. F. 1991, A\&AS, 91, 285

Thuan, T. X., Lipovetsky, V. A., Martin, J.-M., \& Pustilnik, S. A. 1999, A\&AS, 139, 1

Tikhonov, N. A., Galazutdinova, O. A., \& Drozdovsky, I. O. 2000, Astrofiz., 43, 503

Ugryumov, A. V., Engels, D., Kniazev, A. Y., et al. 2001, A\&A, 374,907

van Zee, L., Haynes, M. P., \& Giovanelli, R. 1995, AJ, 109, 990

Zasov, A., Kniazev, A., Pustilnik, S., et al. 2000, A\&AS, 144, 429 